\title{
Malas noticias para el Plan AUGE
}

\author{
Bad news for the AUGE
}

En el reciente discurso del 21 de mayo, el Presidente Piñera ha ratificado su compromiso con subir a 80 los problemas de salud de los chilenos, que están cubiertos en las Garantías Explícitas de Salud (GES), mejor conocidas como AUGE, a partir de Julio de este año 2013. Sin embargo, lo que parece ser una muy buena noticia para el común de nuestros ciudadanos e inclusive para los médicos y profesionales de la salud de nuestro país, en realidad es más bien una pésima señal y muestra cómo los dos últimos gobiernos, independientemente de su color político, en realidad están matando el AUGE o GES, poniendo en jaque a una de las más exitosas políticas públicas de los últimos 23 años en nuestro país.

Para comprender esta aparente paradoja debemos partir por explicar que las 56 patologías GES en su diseño original fueron priorizadas en función de criterios de magnitud, trascendencia, oferta de tratamientos efectivos, costos catastróficos y carga de enfermedad, es decir,de cuantos chilenos se enferman, mueren o quedan con discapacidad a consecuencia de padecerlas. Junto con ello y solo para el Fondo Nacional de Salud, para el año 2005 se calcularon los costos para su financiamiento, considerando las guías clínicas y sus respectivas canastas de prestaciones en condiciones ideales o de acuerdo a la Lex Artis de la Medicina. Dicho calculo determinó que para cada beneficiario del sector público el financiamiento anual de estas patologías sería de 3,06 UF, a lo que habría que agregar su reajuste en base a la evolución del índice de remuneraciones de los trabajadores del sector privado, calculado por el Instituto Nacional de Estadísticas (INE); todo con el fin de que los recursos considerados, con el paso de los años, no perdiesen su poder de compra de prestaciones en salud. De hecho, aplicado el indicador de reajuste precedente desde el año 2005 hasta este año 2013, ya no son 3,06 UF sino 3,86 UF, los recursos potencialmente disponibles para cada beneficiario.

A lo anterior falta agregar que este diseño teórico o ideal tampoco pudo ser correcta y totalmente implementado, básicamente por los problemas de eficiencia y productividad de nuestros hospitales autogestionados y centros asistenciales, así como por fallas en los sistemas de control y registro clínico asistencial. El Ministerio de Hacienda terminó entonces autorizando menos recursos de los que legal y técnicamente estaba en condiciones de otorgar; de hecho para el año 2007 cuando las patologías incorporadas a las GES ya eran 56, el financiamiento autorizado llegaba aproximadamente a 2,6 UF, así para cuando el gobierno anterior para el año 2010 decidió subir a 69 las patologías, contaba con el financiamiento disponible por dos razones: Primero porque las patologías incorporadas hasta ese momento contaban con canastas de prestaciones suboptimas o sin considerar la etapa diagnóstica o de seguimiento de varias de ellas; y segundo, porque tampoco se habían contabilizado a la fecha los reajustes legales del financiamiento, que debían ser calculados por el INE. Baste recordar que fue el propio Consejo Consultivo del AUGE, el que a fines del año 2009 recomendó al Ministerio de Salud de la época no aumentar el número de patologías, sino que por el contrario sugirió utilizar los recursos disponibles en mejorar las canastas de prestaciones de las 56 ya incorporadas.

Lamentablemente esta razonable sugerencia fue finalmente desestimada por la autoridad ministerial y fue así como se dió inicio a lo que a mi juicio constituye el principio del fin para la correcta implementación del AUGE o GES. En otras palabras, lo que había sido diseñado en su financiamiento para 56 problemas de salud, por las razones expuestas, hoy estaba con los mismos recursos pero para 69.

Pero cuando pensábamos que esta cuestionable práctica no se volvería a repetir, ahora es el gobierno actual el que vuelve tropezar con la misma piedra. Efectivamente desde el año 2012 el actual gobierno ha estado preparándose para subir las GES de 69 a 80 problemas de salud, pero nuevamente solo con los recursos adicionales dados por canastas insuficientes y por los reajustes acumulados no aplicados.

Las consecuencias de estas incorrectas decisiones no se han dejado esperar. En primer lugar la evaluación de la implementación del AUGE o GES ha venido descendiendo gradualmente desde el año 2007 en la 
percepción ciudadana tanto en el sector público como privado. Aunque esta partió el 2005 con un 5,9 (en una escala de 1 a 7) y luego el 2006 y 2007 subió a un 6,0, desde el 2008 ha estado descendiendo para situarse el año 2011 (la última disponible) en un solo regular 5,4. Vale destacar en este punto que el propio gobierno, consciente de estas malas evaluaciones, decidió lisa y llanamente no hacer una evaluación el año 2012, por lo que no tenemos disponible a estas alturas un seguimiento actualizado de esta negativa tendencia.

En segundo lugar, el hecho de que ahora tengamos 80 patologías ahi donde el diseño original de su financiamiento solo permitía 56, ha redundado en que algunos de los nuevos problemas de salud tampoco contengan canasta de prestaciones para el diagnóstico, tratamiento y seguimiento de varias de ellas o que en su defecto las que se han considerado no contengan las prestaciones que han sido recomendadas por las sociedades cientificas. El caso que ilustra esta situación ha sido el de la incorporación del Cáncer Colorectal para el último decreto GES. En efecto, si bien la guía clínica recoge la mayor parte de las recomendaciones de los especialistas, la canasta de prestaciones garantizada adolece de graves insuficiencias, la más notoria es la eliminación casi completa de todas las prestaciones que se requieren para tratar los canceres de ubicación rectal. Aunque esta situación ha sido asumida por algunas autoridades intermedias del Ministerio de Salud como un lamentablemente error, llama la atención la falta de rigurosidad en tan importante proceso regulatorio de cobertura de beneficios en salud para más de 13 millones de chilenos, ya que su corrección si es que las decisiones se tomaron adecuadamente, pasan o por subir el marco del financiamiento para poder incorporar las prestaciones desestimadas o la bajada de otras canastas o prestaciones de otras patologías previamente aprobadas, ya que el marco de financiamiento no permite su ampliación.

Cierro estas líneas con la convicción de que si no somos capaces a la mayor brevedad posible, de corregir la situación descrita, estaremos conscientemente matando el AUGE o GES, la que en sus inicios se constituyó en una de las mejores políticas públicas de salud de las últimas décadas en nuestro país. Lo primero que habría que hacer, dado que resultaría impracticable políticamente la disminución de patologías ya garantizadas, sería el envío de un proyecto de ley de un solo artículo, en cuyo enunciado se sustituyera el guarismo de 3,06 UF por un nuevo valor, que según los cálculos que hemos hecho en el Instituto de Salud Pública de la Universidad Andrés Bello sería de 4,5 UF; a continuación sería necesario repensar la institucionalidad con que se definen los beneficios y a no solo del GES sino de los aranceles de Fonasa y crear, haciendo un benchmark, el Instituto Nacional de Excelencia Clínica, con sus siglas NICE en inglés, solo así podremos aprender de nuestros errores y retomar la senda de un instrumento de planificación y priorización sanitaria riguroso, que pueda seguir brindando beneficios y mejoras evidentes en acceso y protección financiera a nuestra población.

Dr. Manuel Inostroza P.

Magíster Salud Pública John Hopkins University. Presidente del Consejo Académico del Instituto de Salud Pública de la Universidad Andrés Bello, Santiago, Chile 